\title{
Study on the Mixed Prediction Method of Order Quantity Based on Grey Neural Network
}

\author{
Zhou Yewang
}

\begin{abstract}
Order quantity prediction in modern enterprise management play an very important position, order quantity is influenced by many uncertain factors. the paper put forward a mixed prediction method to predict the order quantity based on grey prediction and artificial neural network, the mixed prediction method can make full use of each single prediction model, can better improve the prediction accuracy. The method is based on grey prediction model, using the advantages simple algorithm less data and BP neural network to nonlinear system prediction performance advantages, the use of BP nonlinear mapping of neural network mixed prediction.
\end{abstract}

Index Terms-Mixed prediction, grey prediction, neural network, order quantity.

\section{INTRODUCTION}

About the order quantity forecasting has been a lot of research in this area, but many are using single forecasting method. Single prediction methods are not very high accuracy, for now the order quantity prediction, it is difficult to achieve accuracy requirements. Betes and Granger published" forecast" in an article that 2or more than 2 kinds of unbiased individual forecast combinations can be better than each single prediction results, so as to effectively improve the accuracy of prediction [1], [2]. The traditional combination forecasting using linear combination method, such as the gray prediction method, extrapolation technique and artificial neural network prediction method for the linear combination of three kinds of prediction. Prediction model for the optimal weights are determined by the least squares, the method based on gradient optimization principle, it is easy to fall into local minima, can not effectively overcome in the modeling of nonlinear and discontinuous, when various predictive value between real value and presented to the linear relations, using method of least squares to get the results will generate large deviation [3], [4]. Based on this, this paper presents a grey combination forecasting model of neural network.

\section{GREY PREDICTION MODEL}

Order quantity is restricted by many factors, such as market, competition, product quality and product life cycle stages and other factors. Therefore it is very difficult to grasp the various factors on the orders. In this respect, order

Manuscript received June 23, 2014; revised August 29, 2014.

Zhou Yewang is with the School of Business, Huanggang Normal University, Huanggang, 438000, China (e-mail: whuw@ sina.com). quantity decision is a grey system, a lot of information is uncertain, we firstly use the grey prediction model (GM) to predict the next phase of the order quantity using past orders. A general modeling is the original data sequence to establish a differential equation; grey modeling is the use of original data sequence generation number after the establishment of differential equation [5].

\section{A. Data Processing}

If given original data series $x^{(0)}=\left(x^{(0)}(1), x^{(0)}(2), \cdots, x^{(0)}(n)\right)$, These data are irregular, random, have obvious swing, if accumulation the original data and generate new data column, $x^{(1)}=\left(x^{(1)}(1), x^{(1)}(2), \cdots, x^{(1)}(n)\right)$, in the above equation, $x^{(1)}(i)=\sum_{k=1}^{i} x^{(0)}(k), i=1,2, \cdots n$. The new generation of data as a monotonic growth curve, increased the original data regularity, and weaken the fluctuation.

\section{B. Build Differential Equations}

Grey system modeling is applied directly to the time series into a differential equation, namely, grey dynamic model the abbreviation is $\operatorname{GM}$, GM $(1,1)$ that contains a variable differential equation of first order dynamic model, GM $(1,1)$ model of first order differential equation is:

$$
\frac{d X^{(1)}(t)}{d t}+a X^{(1)}(t)=u
$$

\section{Estimate Parameter $a A$ and $U$}

Use the least square method we can get

$$
\hat{a}=\left|\begin{array}{l}
a \\
u
\end{array}\right|=\left(B^{T} B\right)^{-1} B^{T} X_{n},
$$

In the equation,

$$
\begin{gathered}
B=\left|\begin{array}{cc}
-1 / 2\left(X^{(1)}(1)+X^{(1)}(2)\right. & 1 \\
-1 / 2\left(X^{(1)}(2)+X^{(1)}(3)\right. & 1 \\
\cdots & \cdots \\
-1 / 2\left(X^{(1)}(n-1)+X^{(1)}(n)\right. & 1
\end{array}\right| \\
X_{n}=\left|\begin{array}{c}
X^{(0)}(2) \\
X^{(0)}(3) \\
\cdots \\
X^{(0)}(n)
\end{array}\right|
\end{gathered}
$$




\section{A Derivative of $G M(1,1)$ Prediction Model}

$$
X^{(1)}(K+1)=\left(X^{(1)}(0)-u / a\right) e^{-a k}+u / a
$$

\section{BP NeURAL NeTWORK PREDICTION MODEL}

At present, in the applications of the artificial neural network, the vast majority of neural network models are used in the error back propagation learning algorithm of feedforward neural network. BP neural network algorithm is as follows: The guiding ideology of BP neural network is: correct network weights $\left(w_{i j}, T_{l i}\right)$, and threshold $(\theta)$, make difference function ( $\mathrm{E}$ ) drop along the negative gradient direction, Three layer of BP network nodes is represented as, input node: $x_{j}$, implicit node $y_{i}$, the output node $o_{l}$, the weights of the network between the output node and hidden nodes $w_{i j}$, the weights between hidden nodes and the output node $T_{l i}$, the desired output the of output node is $t_{l}$, The basic calculation formula of BP algorithm is as follows [6], [7]:

\section{A. The Output Node Calculation Formula}

The hidden node output

$$
y_{i}=f\left\{\sum_{j} w_{i j}-\theta_{i}\right\}=f\left(n e t_{i}\right)
$$

The output of output node

$$
o_{l}=f\left\{\sum_{i} T_{l i} y_{i}-\theta_{l}\right\}=f\left(\text { net }_{l}\right)
$$

\section{B. Transfer Function $f(x)$ Derivative Formula}

Function

$$
f(x)=\frac{1}{1+e^{-x}}
$$

The relationship between

$$
f^{\prime}(x)=f(x)(1-f(x))
$$

Then

$$
f^{\prime}\left(n e t_{k}\right)=f\left(n e t_{k}\right)\left(1-f\left(n e t_{k}\right)\right)
$$

Output node

$$
f^{\prime}\left(n e t_{1}\right)=o_{l}\left(1-o_{l}\right)
$$

Hidden node

$$
f^{\prime}\left(\text { net }_{i}\right)=y_{i}\left(1-y_{i}\right)
$$

\section{The Output Layer Correction Formula}

Error control:

$$
E=\sum_{k=1}^{p} e_{k}<\varepsilon
$$

Error formula

$$
\partial_{1}=\left(t_{l}-o_{l}\right) o_{l}\left(1-o_{l}\right)
$$

Weighting formula

$$
T_{l i}(k+1)=T_{l i}(k)+\eta \partial_{l} y_{i}
$$

Threshold formula

$$
\theta_{l}(k+1)=\theta_{l}(k)+\eta \partial_{l}
$$

\section{BASED ON THE GRAY GM (1.1) MODEL AND BP NEURAL NETWORK COMBINATION FORECAST MODEL}

The paper combine two forecasting methods advantages and build combination forecast model of the order quantity based on Grey Theory and neural network. Using neural networks to modified residuals of grey model part use gray model to correct neural network prediction error. This model not only has advantages which GM (1.1) model grasp data uncertainty, but also the integration of artificial neural network in the prediction of field edge of uncertain factors, Avoid GM (1.1) model with exponential curve to simulate the instrument prediction, exponential curve is easy to occur the shortcomings such as the seasonal fluctuation is large, some accidental factor data fitting poor.

The order quantity based on grey neural network combination forecast model step [8]:

1) Input historical data series of order amount

2) Predict the data series using grey model and neural network model

3) select training samples Grey Optimal GM (1.1) model prediction value and pure neural network prediction value as a combination input, the actual order series as the output of the neural network, train neural network, determine the weights and threshold.

4) Use the selected samples of the grey model prediction value and pure neural network predictive value as a combination input of the neural network, use the trained neural network training, the combination forecasting value.

\section{EXAMPLE}

In this section, we use an example to validate the superiority of the combination forecasting model. We use the grey prediction method and pure neural network prediction method of combination forecast model as order quantity in the single prediction method, then the network input layer neuron number is determined as 2 , after operation test, the number of hidden layer neurons was determined to be 4 , the input layer neuron number is BP neural network learning sample is composed by input and output sample. The input samples is by orders of combination forecasting model selected two forecasting methods, combine the various moments order quantity forecast, constitute the input of BP neural network sample matrix. Set a company recently some items in order for ten months data constitute the input of BP neural network sample, will last ten months actual orders as the neural network output sample. According to the calculation method can get three kinds of prediction method and its relative error comparison results are shown in Table I: 
TABLE I: COMPARISON OF PREDICTION RESULTS OF ORDERS QUANTITY

\begin{tabular}{|c|c|c|c|c|c|c|c|}
\hline Month & $\begin{array}{c}\text { Practical } \\
\text { value }\end{array}$ & $\begin{array}{c}\text { Pure neural } \\
\text { network } \\
\text { predictive } \\
\text { value }\end{array}$ & $\begin{array}{l}\text { Relative } \\
\text { error\% }\end{array}$ & $\begin{array}{l}\text { Grey model } \\
\text { prediction } \\
\text { value }\end{array}$ & $\begin{array}{l}\text { Relative } \\
\text { error\% }\end{array}$ & $\begin{array}{l}\text { Grey neural } \\
\text { network } \\
\text { prediction }\end{array}$ & $\begin{array}{r}\text { Relative } \\
\text { error\% }\end{array}$ \\
\hline 1 & 999 & 1003 & 0.4 & 999 & 0 & 982 & -1.65 \\
\hline 2 & 1334 & 1303 & -2.32 & 1360 & 1.95 & 1357 & 1.74 \\
\hline 3 & 1523 & 1578 & 3.61 & 1512 & -0.72 & 1537 & 0.94 \\
\hline 4 & 1718 & 1714 & -0.23 & 1680 & -2.16 & 1706 & -0.67 \\
\hline 5 & 1846 & 1796 & 2.72 & 1869 & 1.22 & 1829 & -0.94 \\
\hline 6 & 1930 & 1925 & -0.26 & 2077 & 7.63 & 1912 & -0.95 \\
\hline 7 & 2124 & 2182 & 2.73 & 2309 & 8.82 & 2143 & 0.89 \\
\hline 8 & 2669 & 2645 & -0.9 & 2567 & -3.81 & 2661 & -0.28 \\
\hline 9 & 3088 & 2892 & -6.35 & 2854 & -7.57 & 3067 & -0.68 \\
\hline 10 & 3324 & 3167 & -4.72 & 3152 & -5.18 & 3358 & 1.01 \\
\hline
\end{tabular}

In order to further test the combination forecasting result is good or bad, must develop a set of feasible evaluation index on combination forecasting effect for a full range of integrated measurement and evaluation. This paper adopts the following evaluation index.

\section{A. Sum of Square Error}

$$
S S E=\sum_{i=1}^{n}\left(y_{i}-y_{i}^{\prime}\right)^{2}
$$

On the type, $y_{i}$ predict the actual value, $y_{i}^{\prime}$ is the predictive value.

\section{B. The Mean Absolute Error}

$$
M A E=\frac{1}{n} \sum_{i=1}^{n}\left|y_{i}-y_{i}^{\prime}\right|
$$

\section{The Mean Error}

$$
M S E=\frac{1}{n} \sum_{i=1}^{n}\left(y_{i}-y_{i}^{\prime}\right)^{2}
$$

The results of the prediction error index are shown in Table II:

TABLE II: THE PREDICTION ERROR INDEX

\begin{tabular}{|c|c|c|c|}
\hline & SSE & MAE & MSE \\
\hline Pure neural network model & 7.35 & 0.58 & 0.74 \\
GM (1, 1) prediction model & 15.33 & 0.94 & 1.53 \\
$\begin{array}{c}\text { Grey neural network } \\
\text { prediction model }\end{array}$ & 0.38 & 0.18 & 0.04 \\
\hline
\end{tabular}

From the table we can see the results of the forecast analysis based on artificial neural network, nonlinear combination forecasting method can get better effect than the original single forecasting method, the error index are lower than the single forecasting error indicator, examples show the rationality and feasibility of the method to predict the order quantity.

\section{CONCLUSION}

Accurate prediction of order quantity can make enterprises in the fierce competition in the market to reduce the risk, can make the enterprise products occupy the market quickly. This paper attempts to use the gray forecasting model and the neural network prediction model of combination forecasting. Finally, through an example to illustrate the method can effectively improve the prediction accuracy, reduce the prediction error. Based on the grey theory and neural network's order volume combination forecasting model is proposed, extract the useful part of each single forecasting model, But implementation of the combination forecasting model is affected by many factors, how to improve the neural network's learning effect, determine the comprehensive prediction of nonlinear function and how to avoid every single forecasting model provides a valid information, are in need of further study. Moreover, the method for short-term prediction is more effective, high precision, but for long term prediction is not necessarily can achieve satisfactory results, this is the future in the study of combination forecasting to the direction of learning.

\section{REFERENCES}

[1] H. S. Kim, R. Eykholt, and J. D. Salas, "Nonlinear dynamicsdelaytimes and embedding windows," Physica D., no. 127, pp. 48-60, 1999.

[2] K. C. Luk, J. E. Bal, and A. Sharma, "A study of optimalmodellag and spatial inputs to artificialneuralnetwork for rainfall forecas-ting," Journal of Hydrology, no. 227, pp. 56-65, 2000.

[3] G. B. Zhao, Y. F. Shi, and W. F. Duan et al., "Computing fractaldimension and the kolmogorov entropy fromchaotic time 
series," Chinese Journal of Computation Physics, vol. 16, no. 3, pp. 309-315, 1999

[4] J. H. Lv, J. A. Lu, and S. H. Chen, Chaotic Time Series Analysis and It'S Applications, WuHan: WuhanUniversity Press, 2002.

[5] K. Chen and B. T. Han, "A survey of state space re-construction of chaotic time series analysis," Computer Science, vol. 32, no. 4, pp. $67-70,2005$.

[6] X. H. Wen and M. H. Niu, "A neural network based nonlinear combination forecasting method," System Engineering Theory and Practice, vol. 12, 1994.

[7] Q. Zhang, "Study on the Neural network based optimal combination forecasting method," System Engineering Theory and Practice, vol. $9,2001$.
[8] T. Luo, "Optimization grey model," Chinese Academy of Engineering, vol. 15, 2003.

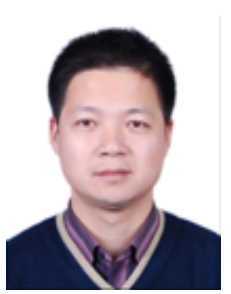

Yewang Zhou was born in Hubei Province, China in July, 1974. He received the Ph.D. degree of engineering, graduated from School of Transportation, Wuhan University of Technology. And his research interests are intelligent control and computer application technology.

$\mathrm{He}$ has been an associate professor of School of Business, Huanggang Normal University. 